Bull. Austral. Math. Soc.

VOL. 39 (1988) [351-356]

\title{
A CLASS OF FUNCTIONAL EQUATIONS WHICH HAVE ENTIRE SOLUTIONS
}

\author{
Peter L. Walker
}

We consider the Abelian functional equation

$$
g(\phi(z))=g(z)+1
$$

where $\phi$ is a given entire function and $g$ is to be found. The inverse function $f=g^{-1}$ (if one exists) must satisfy

$$
f(w+1)=\phi(f(w)) \text {. }
$$

We show that for a wide class of entire functions, which includes $\phi(z)=e^{z}-1$, the latter equation has a non-constant entire solution.

\section{INTRODUCTION}

A functional equation of the form

$$
g(\phi(z))=g(z)+1
$$

where $\phi$ is given, and $g$ is to be found, is said to be of Abelian type, following the paper of Abel [1].

The inverse function $f=g^{-1}$ satisfies

$$
f(w+1)=\phi(f(w))
$$

where we have put $w=g(z)$.

Solutions of these equations are of importance in studying the flow in a set $X$ determined by a map $\phi$ of $X$ to itself, since the family of functions

$$
\phi_{t}(z)=f(g(z)+t), \quad t \in \mathbf{R}
$$

satisfies the formal identities

$$
\phi_{0}(z)=z, \phi_{1}(z)=\phi(z), \text { and } \phi_{t}\left(\phi_{u}(z)\right)=\phi_{t+u}(z)
$$

When $X=C$ and $\phi$ is entire, there are obvious difficulties in the analytic continuation of solutions of (1) because of the complicated nature of the singularities of $g$

Received 1 February 1988

Copyright Clearance Centre, Inc. Serial-fee code: 0004-9729/88 \$A2.00+0.00. 
which occur near any fixed point of $\phi$. By contrast we show in this paper that for a reasonably wide class of entire functions $\phi$, the equation (2) has an entire solution: the result is stated as Theorem 2 below.

An important special case is given by $\phi_{0}(z)=e^{z}-1$. Solutions $g_{0}(z)$ of $(1)$ are constructed in [3] for real positive argument, and in [4] for certain regions in $C$. Some other special cases where one can give explicit solutions of (2) are given for constants $a, c>1$, by $\phi(z)=c z, f(z)=c^{z}$, and by $\phi(z)=z^{c}, f(z)=a^{c^{z}}$. These illustrate the general situation in which solutions of $(2)$ tend to increase much more rapidly than $\phi$ itself.

\section{Construction of Solutions}

We begin by stating the following important theorem of Fatou.

Theorem A. (Fatou [2]) Let $\phi(z)=z+\sum_{n=1}^{\infty} c_{n} z^{n+1}$ be an entire function with $c_{1}>0$, and let $N$ be a neighbourhood of 0 on which $\phi$ is invertible.

Then there is an open subset $S$ of $N$ with the following properties:

(i) the origin is a boundary point of $S$, and $(0, t) \subseteq S$ for some $t>0$;

(ii) $\phi^{-1}(S) \subseteq S$;

(iii) if for any $z \in S$, we put $z_{0}=z, z_{n+1}=\phi^{-1}\left(z_{n}\right), n \geqslant 0$, then we have the asymptotic expansion

$$
\frac{1}{z_{n}}=a n+b \log n-a g(z)+0\left(\frac{\log n}{n}\right) .
$$

In (3) we have $a=c_{1}, b=\frac{1}{c_{1}}\left(c_{2}-c_{1}^{2}\right)$, and $g$ is an analytic function on $S$ which satisfies $g\left(\phi^{-1}(z)\right)=g(z)-1$ for all $z \in S$. The order term is uniform on compact subsets of $S$.

Note:. Fatou proves the result in much greater generality: the above is sufficient for our needs. One can be more explicit about the set $S$, whose boundary is the image of a parabolic arc $x+y^{2}=$ constant $(>0)$ under the inversion mapping $z \rightarrow \frac{1}{z}$; thus the boundary of $S$ is tangent to the negative real axis at the origin.

We can now state our method for constructing solutions of (2). Equation (3) defines $g(z)$ as a limit

$$
\begin{aligned}
w=g(z) & =\lim _{n \rightarrow \infty}\left[n+\frac{b}{a} \log n-\left(a z_{n}\right)^{-1}\right] \\
& =\lim _{n \rightarrow \infty}\left[n+\frac{b}{a} \log n-\left\{a\left(\phi^{-1}\right)^{[n]}(z)\right\}^{-1}\right] \\
& =\lim _{n \rightarrow \infty} g_{n}(z) \text { say. }
\end{aligned}
$$


(For any function, we use $f^{[n]}$ to denote the $n$-fold iterate of $f$.)

We invert this relation to get

$$
z=f(w)=g^{-1}(w)=\lim _{n \rightarrow \infty} g_{n}^{-1}(w)=\lim _{n \rightarrow \infty} \phi^{[n]}\left(\{a(n-w)+b \log n\}^{-1}\right)
$$

Thus our aim is to show the existence of the limit in (4) for all $w \in \mathbb{C}$, which then defines a non-constant entire solution of (2).

We begin with the following result.

TheOREM 1. Let $\phi$ be an entire function of the form $\phi(z)=z+\sum_{1}^{\infty} c_{n} z^{n+1}$, with $c_{1}>0$ and $c_{n} \geqslant 0$ for $n \geqslant 2$. Put $a=c_{1}, b=\frac{1}{c_{1}}\left(c_{2}-c_{1}^{2}\right), r_{n}=n+\frac{b}{a} \log n$, and define

$$
f_{n}(w)=\phi^{[n]}\left(\{a(n-w)+b \log n\}^{-1}\right) .
$$

Then $f_{n}$ is analytic on $C \backslash\left\{r_{n}\right\}$, and for any $M>0$, the sequence $\left(f_{n}\right)_{r_{n}>M}$ is uniformly bounded on $\bar{S}(0, M)=\{z:|z| \leqslant M\}$, provided that either (i) $c_{2} \neq c_{1}^{2}$, or (ii) $c_{3}<c_{1}^{3}$.

Proof: Since $c_{n} \geqslant 0$ for all $n$, the Maclaurin coefficients of $f_{n}$ are also nonnegative. In particular for $|w|<r_{n}$, we have $\left|f_{n}(w)\right| \leqslant f_{n}(|w|)$. Thus it will be sufficient to show that the sequence $\left(f_{n}(w)\right)$ is convergent for $w>0$. In fact we shall show, subject to either of the conditions (i) or (ii), that for $w>0$ the sequence $\left(f_{n}(w)\right)$ is eventually decreasing. Since $\phi$ is monotone increasing on $[0, \infty)$ it is sufficient to prove, for $w>0$ and sufficiently large $n$, that

$$
\phi\left(\alpha_{n}\right)<\alpha_{n-1}
$$

where we have put $\alpha_{n}=\{a(n-w)+b \log n\}^{-1}$.

To prove (5), we expand both sides asymptotically and compare terms. For ease of calculation, we put $k=\frac{b}{a}$, and $w_{n}=w-k \log n$, so that $\alpha_{n}=\frac{1}{a\left(n-w_{n}\right)}$, and the result to be proved is that

$$
\begin{aligned}
& \alpha_{n-1} / \alpha_{n}>\phi\left(\alpha_{n}\right) / \alpha_{n}=1+\sum_{1}^{\infty} c_{r}\left(\alpha_{n}\right)^{r} \quad \text { or } \\
& \frac{n-w_{n}}{n-1-w_{n-1}}>1+c_{1} \alpha_{n}+c_{2} \alpha_{n}^{2}+\ldots
\end{aligned}
$$

Now $w_{n-1}=w-k \log (n-1)=w-k \log n-k \log \frac{n-1}{n}=w_{n}+k s_{n}$, say, where $s_{n}=-\log \left(\frac{n-1}{n}\right)=\frac{1}{n}+\frac{1}{2 n^{2}}+\ldots=0\left(\frac{1}{n}\right)$. 
Hence on the left hand side of (6) we have

$$
\frac{n-w_{n}}{n-w_{n}-1-k s_{n}}=\left(1-\frac{w_{n}}{n}\right)\left(1-\frac{w_{n}+1+k s_{n}}{n}\right)^{-1}
$$

which we expand as far as terms in $n^{-3}$, to get

$$
\begin{aligned}
\left(1-\frac{w_{n}}{n}\right) & {\left[1+\frac{1}{n}\left(w_{n}+1+k s_{n}\right)+\frac{1}{n^{2}}\left(\left(w_{n}+1\right)^{2}+2 k s_{n}\left(w_{n}+1\right)\right)\right.} \\
& \left.+\frac{1}{n^{3}}\left(w_{n}+1\right)^{3}+0\left(\left(\frac{\log n}{n}\right)^{4}\right)\right] \\
& =1+\frac{1}{n}+\frac{1}{n^{2}}\left\{\left(w_{n}+1\right)^{2}-w_{n}\left(w_{n}+1\right)\right\}+\frac{k}{n} s_{n} \\
& +\frac{1}{n^{3}}\left\{\left(w_{n}+1\right)^{3}-w_{n}\left(w_{n}+1\right)^{2}\right\}-\frac{k}{n^{2}} s_{n} w_{n}+\frac{2 k}{n^{2}} s_{n}\left(w_{n}+1\right) \\
& +0\left(\left(\frac{\log n}{n}\right)^{4}\right) \\
& =1+\frac{1}{n}+\frac{1}{n^{2}}\left(w_{n}+1\right)+\frac{k}{n}\left(\frac{1}{n}+\frac{1}{2 n^{2}}\right)+\frac{1}{n^{3}}\left(w_{n}+1\right)^{2}-\frac{k w_{n}}{n^{3}} \\
& +\frac{2 k}{n^{3}}\left(w_{n}+1\right)+0\left(\left(\frac{\log n}{n}\right)^{4}\right) \\
& =1+\frac{1}{n}+\frac{1}{n^{2}}\left(w_{n}+1+k\right)+\frac{1}{n^{3}}\left(\left(w_{n}+1\right)^{2}+k\left(w_{n}+\frac{5}{2}\right)\right) \\
& +0\left(\left(\frac{\log n}{n}\right)^{4}\right) .
\end{aligned}
$$

Similarly on the right hand of (6), we substitute $\alpha_{n}=\frac{1}{a\left(n-w_{n}\right)}$ and $c_{1}=a, c_{2}=$ $a^{2}(1+k)$ to get

$(* *)$

$$
\begin{aligned}
1+c_{1} \alpha_{n} & +c_{2} \alpha_{n}^{2}+c_{3} \alpha_{n}^{3}+0\left(\alpha_{n}^{4}\right) \\
& =1+\left(n-w_{n}\right)^{-1}+(1+k)\left(n-w_{n}\right)^{-2}+\frac{c_{3}}{a^{3}}\left(n-w_{n}\right)^{-3}+0\left(n^{-4}\right) \\
& =1+\frac{1}{n}\left(1+\frac{w_{n}}{n}+\frac{w_{n}^{2}}{n^{2}}\right)+\left(\frac{1+k}{n^{2}}\right)\left(1+\frac{2 w_{n}}{n}\right)+\frac{c_{3}}{a^{3} n^{3}}+0\left(\frac{(\log n)^{3}}{n^{4}}\right) \\
& =1+\frac{1}{n}+\frac{1}{n^{2}}\left(w_{n}+1+k\right)+\frac{1}{n^{3}}\left(w_{n}^{2}+2(1+k) w_{n}+\frac{c_{3}}{a^{3}}\right)+0\left(\frac{(\log n)^{3}}{n^{4}}\right) .
\end{aligned}
$$


If we compare $\left(^{*}\right)$ and $\left({ }^{* *}\right)$ we see that $(6)$ is equivalent (for sufficiently large $n$ ) to the inequality

$$
\left(w_{n}+1\right)^{2}+k\left(w_{n}+\frac{5}{2}\right)>w_{n}^{2}+2(1+k) w_{n}+\frac{c_{3}}{a^{3}}
$$

or to $1+5 k / 2>k(w-k \log n)+\frac{c_{3}}{a^{3}}$.

But this inequality is evidently satisfied for all $n$ sufficiently large, if either (i) $k \neq 0$, (equivalently $c_{2} \neq c_{1}^{2}$ ), or (ii) if $k=0$, then $c_{3}<a^{3}=c_{1}^{3}$. Hence either condition (i) or (ii) is sufficient to establish (6), which completes the proof of Theorem 1 .

The uniform boundedness which we have just proved enables us to deduce our main theorem on existence of solutions of (2).

TheOREM 2. Let $\phi$ be an entire function of the form $\phi(z)=z+\sum_{1}^{\infty} c_{n} z^{n+1}$, where $c_{1}>0, c_{n} \geqslant 0$ for all $n$, and either (i) $c_{2} \neq c_{1}^{2}$ or (ii) $c_{3}<c_{1}^{3}$.

Then the sequence $\left(f_{n}\right)$ defined in Theorem 1 converges uniformly on every $\bar{S}(0, M)$ to a function $f$ which is an entire non-constant solution of (2).

Proof: Theorem 1 shows that the sequence $\left(f_{n}\right)$ forms a normal family on each $\bar{S}(0, M)$. In the course of the proof we also showed that for any $M>0$ and sufficiently large $n$, the restrictions of $f_{n}$ to $[-M, M]$ form a sequence of positive functions which decreases with increasing $n$, and so converges on $[-M, M]$ to a limit $\psi$ say. Hence any subsequence of $\left(f_{n}\right)$ which converges on $\bar{S}(0, M)$ must have a limit which agrees with $\psi$ on the real axis, from which we deduce the convergence of the whole sequence to an entire function $f$, whose restriction to $[-M, M]$ is $\psi$. Moreover, since $f_{n}$ is defined as the inverse of the function $g_{n}$ for which $g_{n}(z) \rightarrow g(z)$ for $z \in S$ (Fatou's Theorem A), $f$ must equal $g^{-1}$, on some open subset $U$, say, of $g(S)$, (for instance a neighbourhood of $g(S \cap(0, \infty)))$, so $f$ cannot be constant. Again since $f=g^{-1}$, we must have (2) at least on $g(S \cap(0, \infty))$. But both sides of (2) are entire, and so the equation must hold generally and the proof of Theorem 2 is complete.

To conclude, we mention some general properties of the function $f$ which we have constructed. Since $f_{n}(w)=\phi^{[n]}\left(\frac{1}{a(n-w)+b \log n}\right)$, and $\phi(t)=t+\sum_{1}^{\infty} c_{n} t^{n}, c_{n} \geqslant 0$, it follows that $f$ is a positive increasing function on $\mathbf{R}$, whose Maclaurin coefficients are again non-negative. We can deduce the asymptotic rate at which $f(x) \rightarrow 0$ as $x \rightarrow-\infty$, from the corresponding expansion for $g(t)$ as $t \rightarrow 0_{+}$, in the following way. First simplify the asymptotic expansion (3) of Fatou's Theorem to read $\frac{1}{t_{n}}=$ $a n+b \log n-a g(t)+o(1)$, for $t>0, t \in S$. The functional equation satisfied by $g$ shows that $g\left(t_{n}\right)=g(t)-n$, and hence if we put $x=t_{n}, y=g\left(t_{n}\right)$ so that $x \rightarrow 0_{+}$, 
$y \rightarrow-\infty$ as $n \rightarrow \infty$, then we obtain

$$
\begin{aligned}
g(x)=g(t)-n & =-\frac{1}{a x}+k \log n+o(1) \\
& =-\frac{1}{a x}+k \log \left(\frac{1}{a x}-k \log n+a g(t)+o(1)\right) \\
& =-\frac{1}{a x}+k \log \left(\frac{1}{a x}\right)+o(1) \text { as } x \rightarrow 0_{+} .
\end{aligned}
$$

Similarly, we can show that $\lim _{x \rightarrow 0_{+}} x^{2} g^{\prime}(x)=\frac{1}{a}$, which is sufficient for the unique determination of a solution of (1) (up to an additive constant), as is pointed out by Szekeres in [3, Lemma 1].

Then the get the asymptotic expansion of $f(x)$ as $x \rightarrow-\infty$, we invert the above expansion for $g$ to obtain

$$
a f(x)=-\frac{1}{x}+\frac{k}{x^{2}} \log |x|+o\left(x^{-2}\right):
$$

in particular $x f(x) \rightarrow-\frac{1}{a}$ as $x \rightarrow-\infty$.

In the special case when $\phi(t)=e^{t}-1$, the hypotheses of Theorem 2 are satisfied and we can deduce the existence of an entire non-constant solution of the equation $f(w+1)=e^{f(w)}-1$. This function is inverse to the function $g$ constructed in [4], Theorem 2, which is analytic on $S=\mathrm{C} \backslash(-\infty, 0]$ and satisfies $g(\log (1+z))=g(z)-1$ for all $z$ in $S$. Hence the family of mappings $\phi_{t}(z)=f(g(z)-t), t \geqslant 0$, determines the flow of the map $z \rightarrow \log (1+z)$ in $S$.

\section{References}

[1] N.H. Abel, 'Determination d'une fonction au moyen d'une equation qui ne contient qu'une seule variable', in Oeuvres Completes de Niels Henrik Abel II, p. 246 (Christiana Impr. de Grondahl, 1881).

[2] P. Fatou, 'Sur les equations fonctionelles', Bull. Soc. Math. France 47 (1919), 161-271.48, (1920) pp. 33-94, 208-314.

[3] G. Szekers, 'Fractional iteration of expronentially growing functions', J. Austral. Math. Soc. 2 (1961-2), 301-320.

[4] P.L. Walker, 'On the solutions of an Abelian functional equation', (Submitted).

Department of Mathematical Sciences

Sultan Qaboos University

P.O. Box 32486, Al-Khoudh

Muscat

Sultanate of Oman 